\title{
Actions of certain arithmetic groups on Gromov hyperbolic spaces
}

\author{
JASON FOX MANNING
}

\begin{abstract}
We study the variety of actions of a fixed (Chevalley) group on arbitrary geodesic, Gromov hyperbolic spaces. In high rank we obtain a complete classification. In rank one, we obtain some partial results and give a conjectural picture.
\end{abstract}

20F65; 20E08, 53C24

\section{Introduction}

Given a group $G$ one may ask the question:

Question 1.1 In what ways can $G$ act nontrivially on a Gromov hyperbolic metric space?

Many interesting groups can be fruitfully studied via some natural action on a Gromov hyperbolic space. Examples include the action of an amalgam or HNN extension on its Bass-Serre tree, the action of a Kleinian group on $\mathbb{H}^{n}$ and the action of the mapping class group of a surface on the curve complex of that same surface. Alternatively, one can study the space of actions of a fixed group on some (fixed or varying) Gromov hyperbolic metric space. For example, the $\operatorname{PSL}(2, \mathbb{C})$-character variety of a group parameterizes the space of actions of a fixed group on the hyperbolic space $\mathbb{H}^{3}$. Analysis of the structure of this variety has led to many remarkable theorems about 3-manifolds and their fundamental groups (for an introduction, see Shalen [25]). A larger "variety" (in scare quotes because there is unlikely to be any algebraic structure) would describe all nontrivial actions on Gromov hyperbolic spaces, up to some appropriate equivalence relation. We give suggestions for how to define this equivalence relation and topologize the variety in Section 3. Briefly, the equivalence relation is that generated by coarsely equivariant quasi-isometric embeddings. This equivalence is coarser than that given by quasi-conjugacy (as in for instance Mosher, Sageev and Whyte [23]), but finer than that given by equivariant homeomorphism of limit sets. In this paper we concentrate on cases in which the set of equivalence classes is particularly simple. 
Certain equivalence classes of actions on hyperbolic spaces cannot be ruled out, or even really analyzed using the tools of negative curvature. These are the actions with an invariant horoball (see Section 3.1 for the definition, and Theorem 4.11 for some characterizations). Actions with an invariant horoball (in the sense used in this paper) are always elementary; they include the trivial action on a point and actions which preserve some horofunction. A cobounded action on an unbounded space never has an invariant horoball.

The variety of actions of an irreducible higher rank lattice is expected to be very simple.

Conjecture 1.2 If $\Gamma$ is an irreducible lattice in a higher rank Lie group (or in a nontrivial product of locally compact groups) $G$, there are finitely many Gromov hyperbolic $G$-spaces (up to coarse equivalence) without invariant horoballs.

In the case where $G$ is a simple Lie group of rank at least 2 , the only expected actions are those with an invariant horoball. If $G$ has more than one direct factor, then $\Gamma$ projects densely to each factor. If the factors have rank one, then there will clearly be nonelementary isometric actions of $\Gamma$ on rank one symmetric spaces. We discuss this case in Section 6.

For actions by lattices in nontrivial products of simple Lie groups, the conjecture follows from rigidity results of Monod [20] and Monod-Shalom [21] if one restricts attention to Gromov hyperbolic spaces which are also either CAT(0) spaces, proper and cocompact spaces, or bounded valence graphs. (cf Gelander, Karlsson and Margulis [11] for CAT(0) spaces.) For an example of an action of a lattice on a Gromov hyperbolic space which is inequivalent to any action on a Gromov hyperbolic CAT(0) space, consider a lattice as in the Appendix to our paper [18], which has Property (T), but admits a nontrivial pseudocharacter (or homogeneous quasi-morphism). This pseudocharacter gives rise to a cobounded action on a space quasi-isometric to $\mathbb{R}$, fixing both ends. On the other hand, an action on a CAT(0) space preserving a point at infinity would give (via the Busemann function) a homomorphism to $\mathbb{R}$, and a cobounded such action would give an unbounded homomorphism to $\mathbb{R}$. Such a homomorphism is ruled out by Property (T).

Theorem 1.3 Suppose that $G$ is a simple Chevalley-Demazure group scheme of rank at least 2 , and let $\mathcal{O}$ be the ring of integers of any number field. Then any isometric action of $G(\mathcal{O})$ on a Gromov hyperbolic geodesic metric space has an invariant horoball. 
Some remarks:

(1) Some closely related results are proved by Karlsson and Noskov [15, Sections 8.2 and 8.3]; part of our strategy is similar to theirs, and to that of Fukunaga in [10].

(2) The rank $\geq 2$ assumption is necessary, as the action of $S L(2, \mathcal{O})$ on $\mathbb{H}^{3}$ obtained from the inclusion $S L(2, \mathcal{O}) \longrightarrow S L(2, \mathbb{C})$ never has an invariant horoball.

The rank one case is discussed in Section 6, where we apply a result of Carter, Keller and Paige to show a weaker theorem for these groups.

Theorem 1.4 Let $\mathcal{O}$ be the ring of integers of a number field, and suppose that $\mathcal{O}$ has infinitely many units. Suppose $X$ is quasi-isometric to a tree. Every action of $S L(2, \mathcal{O})$ on $X$ has a bounded orbit.

\subsection{Relative hyperbolicity and bounded generation}

If $G$ is a finitely generated group, $S$ is a generating set for $G$ and $\mathcal{P}=\left\{P_{1}, \ldots, P_{n}\right\}$ is a collection of subgroups of $G$, then one can form the coned space $C(G, \mathcal{P}, S)$ as follows: Let $\Gamma(G, S)$ be the Cayley graph of $G$ with respect to $S$. The coned space $C(G, \mathcal{P}, S)$ is obtained from $\Gamma(G, S)$ by coning each left coset of an element of $\mathcal{P}$ to a point. (Here the 0 -skeleton of $\Gamma(G, S)$ is implicitly identified with $G$.)

Recall that a graph is fine if every edge is contained in only finitely many circuits (ie embedded cycles) of any bounded length.

Definition 1.5 If $G$ is a group with finite generating set $S$ and a collection of subgroups $\mathcal{P}=\left\{P_{1}, \ldots, P_{n}\right\}$, then $G$ is weakly hyperbolic relative to $\mathcal{P}$ if $C(G, \mathcal{P}, S)$ is $\delta$-hyperbolic for some $\delta$.

If, moreover, $C(G, \mathcal{P}, S)$ is fine, then $G$ is (strongly) hyperbolic relative to $\mathcal{P}$.

A special case of weak relative hyperbolicity is bounded generation. The following definition is easily seen to be equivalent to the standard one:

Definition 1.6 A group $G$ is boundedly generated by a collection of subgroups $\mathcal{P}=\left\{P_{1}, \ldots, P_{n}\right\}$ if the Cayley graph of $G$ with respect to $\bigcup \mathcal{P}$ has finite diameter.

If each $P_{i}$ is cyclic, generated by $g_{i}$, we say that $G$ is boundedly generated by $\left\{g_{1}, \ldots, g_{n}\right\}$. 
A cobounded action on an unbounded Gromov hyperbolic space does not have an invariant horoball. It is thus a corollary of Theorem 1.3 that these higher rank $G(\mathcal{O})$ are not strongly relatively hyperbolic with respect to any system of proper subgroups.

Corollary 1.7 If $L=G(\mathcal{O})$ is as in Theorem 1.3, and $L$ is weakly hyperbolic relative to a system of subgroups $\mathcal{P}$, then the coned space $C(L, \mathcal{P}, S)$ has finite diameter for any finite generating set $S$.

In particular, $L$ is not strongly hyperbolic relative to any system of proper subgroups.

By possibly altering $\mathcal{P}$ to add some cyclic subgroups, the first part of the corollary can be restated: If $L$ is weakly hyperbolic relative to a system of subgroups $\mathcal{P}$ which generate $L$, then $L$ is boundedly generated by $\mathcal{P}$.

Remark 1.8 Corollary 1.7 also can be easily deduced from [15] in some special cases, including $L=S L(n, \mathbb{Z})$ for $n>2$. The second part of Corollary 1.7 (about strong relative hyperbolicity) can be deduced from known theorems in a number of ways, perhaps most straightforwardly by combining a theorem of Fujiwara [9] with one of Burger and Monod [4].

\subsection{Outline}

In Section 2 we recall some definitions and basic results, first from the theory of Gromov hyperbolic spaces and second from Chevalley groups over number rings. In Section 3 an equivalence relation amongst hyperbolic $G$-spaces is proposed and generalized combinatorial horoballs are introduced. In Section 4, we improve on the statement and proof of Proposition 3.9 of [18] and use the improved version to characterize hyperbolic $G$-spaces with invariant horoballs. In Section 5 we prove Theorem 1.3, and in Section 6 we prove Theorem 1.4.

Acknowledgments The author thanks Benson Farb and Hee Oh for useful conversations, and Nicolas Monod for helpful comments on an earlier version of this paper. This work was partly supported by an NSF Postdoctoral Research Fellowship (DMS0301954).

\section{Preliminaries}

\subsection{Coarse geometry}

Definition 2.1 If $X$ and $Y$ are metric spaces, $K \geq 1$ and $C \geq 0$, a $(K, C)$-quasiisometric embedding of $X$ into $Y$ is a function $q: X \rightarrow Y$ so that for all $x_{1}, x_{2} \in X$,

$$
\frac{1}{K} d\left(x_{1}, x_{2}\right)-C \leq d\left(q\left(x_{1}\right), q\left(x_{2}\right)\right) \leq K d\left(x_{1}, x_{2}\right)+C .
$$


If in addition the map $q$ is $C$-coarsely onto - ie, every $y \in Y$ is distance at most $C$ from some point in $q(X)$ - then $q$ is called a $(K, C)$-quasi-isometry. The two metric spaces $X$ and $Y$ are then said to be quasi-isometric to one another. This is a symmetric condition.

Definition 2.2 A $(K, C)$-quasi-geodesic in $X$ is a $(K, C)$-quasi-isometric embedding $\gamma: \mathbb{R} \rightarrow X$. We will occasionally abuse notation by referring to the image of $\gamma$ as a quasi-geodesic.

\subsection{Gromov hyperbolic spaces}

For more details on Gromov hyperbolic metric spaces, see Bridson and Haefliger [3] and Gromov [12]. A number of equivalent definitions of Gromov hyperbolicity are known. For geodesic spaces, we will use the one based on the existence of comparison tripods. Given a geodesic triangle $\Delta(x, y, z)$ in any metric space, there is a unique comparison tripod, $T_{\Delta}$, a metric tree so that the distances between the three extremal points of the tree, $\bar{x}, \bar{y}$ and $\bar{z}$, are the same as the distances between $x, y$ and $z$. There is an obvious map $\pi: \Delta(x, y, z) \rightarrow T_{\Delta}$ which takes $x$ to $\bar{x}, y$ to $\bar{y}$ and $z$ to $\bar{z}$, and which is an isometry on each side of $\Delta(x, y, z)$.

Definition 2.3 A geodesic space $X$ is $\delta$-hyperbolic if for any geodesic triangle $\Delta(x, y, z)$ and any point $p$ in the comparison tripod $T_{\Delta}$, the diameter of $\pi^{-1}(p)$ is less than $\delta$. If $\delta$ is unimportant we may simply say that $X$ is Gromov hyperbolic.

Gromov hyperbolicity (of geodesic spaces) is a quasi-isometry invariant.

The following proposition about stability of quasi-geodesics in Gromov hyperbolic spaces is well known (see, eg Bridson and Haefliger [3, III.H.1.7]).

Proposition 2.4 Let $K \geq 1, C \geq 0, \delta \geq 0$. Then there is some $B=B(K, C, \delta)$, so that whenever $\gamma$ and $\gamma^{\prime}$ are two $(K, C)$-quasi-geodesics with the same endpoints in a $\delta$-hyperbolic geodesic metric space $X$, then the Hausdorff distance between $\gamma$ and $\gamma^{\prime}$ is at most $B$.

We will occasionally need to deal with spaces which are not geodesic. If $X$ is $\delta$ hyperbolic in the sense of Definition 2.3, then it satisfies the four point condition: For all $p_{1}, p_{2}, p_{3}, p_{4} \in X$,

$$
\text { (1) } \begin{aligned}
d\left(p_{1}, p_{4}\right)+d\left(p_{2},\right. & \left.p_{3}\right) \\
& \leq \max \left\{d\left(p_{1}, p_{2}\right)+d\left(p_{3}, p_{4}\right), d\left(p_{1}, p_{3}\right)+d\left(p_{2}, p_{4}\right)+2 \delta\right\}
\end{aligned}
$$


Conversely, if a geodesic space satisfies (1), then it is $6 \delta$-hyperbolic in the sense of Definition 2.3. (For both these facts, and the below definition, see Bridson and Haefliger [3, III.H.1.22] or Gromov [12].)

Definition 2.5 A (not necessarily geodesic) metric space $X$ is $(\delta)$-hyperbolic if it satisfies the condition (1) above. If $\delta$ is unimportant, we simply say that $X$ is Gromov hyperbolic.

In order to describe the boundary of a Gromov hyperbolic space, we introduce the Gromov product notation.

Definition 2.6 If $x, y$ and $z$ are points in a metric space with a metric $d(\cdot, \cdot)$, then

$$
(x \cdot y)_{z}:=\frac{1}{2}(d(x, z)+d(y, z)-d(x, y)) .
$$

We should remark that in the context of Definition 2.3, $(x \cdot y)_{z}$ is the distance from $\bar{z}$ to the central vertex of the comparison tripod for a geodesic triangle with vertices $x$, $y$ and $z$.

Definition 2.7 Let $p \in X$ where $X$ is a Gromov hyperbolic space. A sequence of points $\left\{x_{i}\right\}$ in a Gromov hyperbolic space tends to infinity if $\lim _{i, j \rightarrow \infty}\left(x_{i} \cdot x_{j}\right)_{p}=\infty$. Two such sequences are equivalent, written $\left\{x_{i}\right\} \sim\left\{y_{i}\right\}$, if $\lim _{i, j \rightarrow \infty}\left(x_{i} \cdot y_{j}\right)_{p}=\infty$. The boundary $\partial X$ is the set of equivalence classes of sequences which tend to infinity.

The Gromov product extends (by taking a lim sup) to sequences which tend to infinity, and this allows convergence in $\partial X$ to be defined, giving $\partial X$ a natural topology.

\subsection{Isometries of Gromov hyperbolic spaces}

Isometries of geodesic hyperbolic spaces can be classified into three types.

Definition 2.8 Let $f: X \rightarrow X$ be an isometry. If $x \in X$, we let $O_{x}=\left\{f^{n}(x) \mid n \in \mathbb{Z}\right\}$. We say that $f$ is elliptic if $O_{x}$ is bounded. We say that $f$ is hyperbolic if $n \mapsto f^{n}(x)$ is a quasi-isometric embedding of $\mathbb{Z}$ into $X$. We say that $f$ is parabolic if $O_{x}$ has a unique limit point in $\partial G$.

The following was observed by Gromov [12, 8.1.B]. Although it is often stated with an extra hypothesis of properness, this hypothesis is unnecessary (See, for example the proof in [8, Chapitre 9], where the extra hypothesis is given, but not used). 
Proposition 2.9 Every isometry of a geodesic Gromov hyperbolic space is elliptic, parabolic, or hyperbolic.

Lemma 2.10 Suppose that $G$ acts on the geodesic Gromov hyperbolic space $X$, and that $p \in G$ acts parabolically, fixing $e \in \partial X$. If $g \in G$ commutes with $p$, then $g$ also fixes $e$.

Proof Let $e^{\prime}=g(e)$. We have $e^{\prime}=g p(e)=p g(e)=p\left(e^{\prime}\right)$, so $p$ fixes $e^{\prime}$. Since $p$ is parabolic, it fixes a unique point in $\partial X$, and so $e^{\prime}=e$.

Definition 2.11 Let $G$ be a finitely generated group, and let $g \in G$. If $n \mapsto g^{n}$ is a quasi-isometric embedding, we say that $g$ is undistorted. Otherwise, $g$ is distorted.

The proof of the following observation is left to the reader.

Lemma 2.12 Let the finitely generated group $G$ act by isometries on a Gromov hyperbolic space $X$. If $g \in G$ acts hyperbolically on $X$, then $g$ is undistorted.

\subsection{Chevalley groups}

In this section we recall the definition of a Chevalley group over a commutative ring. (All rings are assumed to have $1 \neq 0$.) The simplest example of a Chevalley group is $S L(n, \mathbb{Z})$. If one thinks of $S L(n, \mathbb{Z})$ as being the " $\mathbb{Z}$-points of $S L(n, \mathbb{C})$," then the Chevalley-Demazure group scheme identifies what the " $R$-points of $G$ " are, where $R$ is now allowed to be an arbitrary ring, and $G$ an arbitrary complex semisimple Lie group. It turns out that this idea is not entirely well formed, until one fixes an embedding of $G$ into $G L(n, \mathbb{C})$ for some $n$. The following exposition is largely adapted from Abe [1] and Tavgen' [29].

Let $\rho: G \rightarrow G L(V)$ be a representation of a connected complex semisimple Lie group into the general linear group of a complex vector space $V$ of dimension $n$. We will assume that $d \rho: \mathfrak{g} \rightarrow \mathfrak{g l}(V)$ is faithful (Here $\mathfrak{g}$ is the Lie algebra of $G, \mathfrak{g l}(V)$ the Lie algebra of endomorphisms of $V$. Let $\mathfrak{h}$ be a Cartan subalgebra of $\mathfrak{g}$ and let $\Phi$ be the (reduced) root system relative to $\mathfrak{h}$. Let $\Delta$ be a choice of simple roots. Then there is a Chevalley basis for $\mathfrak{g}$ (see Steinberg [27] or Chevalley [7]) of the form $\mathcal{B}=\left\{X_{\alpha} \mid \alpha \in \Phi\right\} \cup\left\{H_{\alpha} \mid \alpha \in \Delta\right\}$, so that the $H_{\alpha}$ generate $\mathfrak{h}$ (and thus commute) and the structure constants are all integral. In other words, the $\mathbb{Z}$-span of $\mathcal{B}$ is actually a Lie algebra over $\mathbb{Z}$. In particular, if $\left\{\beta-r_{\beta, \alpha} \alpha, \ldots \beta, \ldots \beta+q_{\beta, \alpha} \alpha\right\}$ are all the roots on the line $\{\beta+t \alpha \mid t \in \mathbb{Z}\}$, then:

(1) $\left[X_{\alpha}, X_{-\alpha}\right]=H_{\alpha}$. 
(2) $\left[H_{\alpha}, X_{\beta}\right]=A(\alpha, \beta) X_{\beta}$, where $A(\alpha, \beta)$ is an integer determined by $\alpha$ and $\beta$.

(3) $\left[X_{\alpha}, X_{\beta}\right]=0$ if $\alpha+\beta$ is not in $\Phi$; otherwise $\left[X_{\alpha}, X_{\beta}\right]= \pm\left(r_{\beta, \alpha}+1\right) X_{\alpha+\beta}$.

Notice that the $X_{\alpha}$ are all ad-nilpotent, and that the $H_{\alpha}$ are ad-semisimple.

It can be shown (see, eg Humphreys [14, Section 27]) that $V$ contains an "admissible lattice" $V_{\mathbb{Z}}$ : This is a free $\mathbb{Z}$-module in $V$ which is invariant under $\left(d \rho\left(X_{\alpha}\right)\right)^{m} / m$ ! for any $\alpha$ and any $m$. Let $\left\{v_{1}, \ldots, v_{n}\right\}$ be a basis for $V_{\mathbb{Z}}$. In terms of this basis, $\rho(g)$ can be written as an $n$ by $n$ matrix with complex entries for any $g$. Let $x_{i j}: G \rightarrow \mathbb{C}$ be the function which simply reads off the $i j$-th entry of this matrix. The $x_{i j}$ generate an affine complex algebra $\mathbb{C}(G)$. Let $\mathbb{Z}(G)$ denote the $\mathbb{Z}$-algebra with the same generators.

We can endow $\mathbb{Z}(G)$ with a Hopf algebra structure by defining a comultiplication $\mu^{*}$ by $\mu^{*}\left(x_{i j}\right)=\sum_{k} x_{i k} \otimes x_{k j}$, a counit $\epsilon$ by $\epsilon\left(x_{i j}\right)=\delta_{i j}$, and an antipode $s$ by $s(f)(g)=f\left(g^{-1}\right)$. (That $s$ maps $\mathbb{Z}(G)$ into itself uses the fact that $\operatorname{det}(\rho(g))= \pm 1$ for every $g \in G$ - this follows from semisimplicity.) Since $\mathbb{Z}(G)$ is a Hopf algebra over $\mathbb{Z}$, it defines a functor from rings to groups as follows: For any ring $R$, let $G(R)=$ $\operatorname{Hom}_{\mathbb{Z}}(\mathbb{Z}(G), R)$. (Note that the elements of $G(R)$ are $\mathbb{Z}$-algebra homomorphisms, so they send 1 to 1.) We define a group operation $\bullet$ on $G(R)$ by $\rho \bullet \sigma=(\rho \otimes \sigma) \circ \mu^{*}$. (In other words, $\rho \bullet \sigma\left(x_{i j}\right)=\sum_{k} \rho\left(x_{i k}\right) \sigma\left(x_{k j}\right)$.)

Some observations:

(1) $G(\mathbb{C})$ can be naturally identified with the image of $\rho: G \rightarrow G L(V)-$ if $\rho$ is assumed to be faithful, then clearly $G(\mathbb{C}) \cong G$ doesn't depend on $\rho$. On the other hand, for $R$ arbitrary, $G(R)$ does depend on $\rho$.

(2) The assignment $R \mapsto G(R)$ is a covariant functor from commutative rings to groups. This functor is often called a "Chevalley-Demazure group scheme."

(3) Any Hopf algebra gives such a functor. An important example is $\mathbb{Z}[t]$, with a Hopf ( $\mathbb{Z}-$ )algebra structure so that:

(a) The comultiplication satisfies $\mu^{*}(t)=t \otimes 1+1 \otimes t$.

(b) The counit satisfies $\epsilon(t)=0$.

(c) The antipode satisfies $S(t)=-t$.

In this case the functor $R \mapsto \operatorname{Hom}(Z[t], R)$ is the "forgetful" functor, which takes a ring to its underlying Abelian group.

(4) Since $G(R)$ is $\operatorname{Hom}_{\mathbb{Z}}(\mathbb{Z}(G), R)$, any morphism of Hopf algebras $\mathbb{Z}(G) \rightarrow A$ where $A$ is some other Hopf algebra, will give rise to a homomorphism of groups $\operatorname{Hom}_{\mathbb{Z}}(A, R) \rightarrow G(R)$. 
Definition 2.13 Let $\alpha$ be a root. Then since $d \rho\left(X_{\alpha}\right)$ is nilpotent, the formal sum $\exp \left(t X_{\alpha}\right)=\sum_{m=1}^{\infty} t^{m}\left(d \rho\left(X_{\alpha}\right)^{m} / m !\right)$ is a matrix with entries which are polynomials in $t$. Because $d \rho\left(X_{\alpha}\right)^{m} / m$ ! preserves $V_{\mathbb{Z}}$, each $x_{i j}\left(\left(d \rho\left(X_{\alpha}\right)^{m} / m !\right)\right)$ is an integer. Thus we get a map

$$
\mathrm{ev}_{\alpha}: \mathbb{Z}(G) \rightarrow \mathbb{Z}[t]
$$

which sends $x_{i j}$ to the (integral) polynomial in $t$ which appears in the $i j-$ th place of the matrix $\exp \left(t X_{\alpha}\right)$. The map $\operatorname{ev}_{\alpha}$ is a morphism of Hopf algebras, where $\mathbb{Z}[t]$ is given a Hopf algebra structure as in observation (3) above. This morphism gives rise via observation (4) to a homomorphism of the additive group underlying $R$ into the group $G(R)$

$$
x_{\alpha}: R \rightarrow G(R) .
$$

The image of this map is the root subgroup of $G(R)$ corresponding to $\alpha$.

Definition 2.14 The subgroup of $G(R)$ generated by the root subgroups is denoted $E(R)$.

In this paper, we focus mainly on the special case that $R$ is the ring of integers of a number field $k$ and $\Phi$ has rank at least two. In this case, $G(R)=E(R)$ by a result of Matsumoto [19]. By a result of Carter and Keller [5] in case $\Phi=A_{n}$, and Tavgen' [29] in general, $G(R)$ is boundedly generated by the root subgroups.

It should also be noted that in this special case, $G(R)$ is actually an irreducible lattice in a semisimple Lie group. Indeed, if $s$ and $t$ are the number of real and complex places of $k$, respectively, then $G(R)$ is a lattice in the Lie group

$$
G(\mathbb{R})^{s} \times G(\mathbb{C})^{t} .
$$

\section{Equivalence of actions}

We wish to study the variety of actions of a finitely generated group $G$ on Gromov hyperbolic spaces, up to some kind of coarse equivalence. By a (Gromov) hyperbolic $G$-space, we will always mean a Gromov hyperbolic geodesic metric space, equipped with an isometric $G$-action.

Definition 3.1 Let $X$ and $Y$ be Gromov hyperbolic $G$-spaces. We say that $X$ and $Y$ are equivalent if they lie in the same equivalence class, under the equivalence relation generated by coarsely equivariant quasi-isometric embeddings.

The following proposition should serve to clarify this equivalence relation. 
Proposition 3.2 If $X_{1}$ and $X_{2}$ are equivalent Gromov hyperbolic $G$-spaces, then there is a third Gromov hyperbolic $G$-space $V$ which coarsely equivariantly quasiisometrically embeds in both $X_{1}$ and $X_{2}$.

Proof The key claim is the following:

Claim 3.3 If $V$ and $W$ are hyperbolic $G$-spaces which coarsely equivariantly quasiisometrically embed in a third hyperbolic $G$-space $X$, then there is a fourth hyperbolic $G$-space $A$ which coarsely equivariantly quasi-isometrically embeds into $V$ and $W$.

Before proving the claim, let us see how it implies the proposition. If $X_{1}$ and $X_{2}$ are equivalent hyperbolic $G$-spaces, they must be joined by a sequence of coarsely equivariant quasi-isometric embeddings:

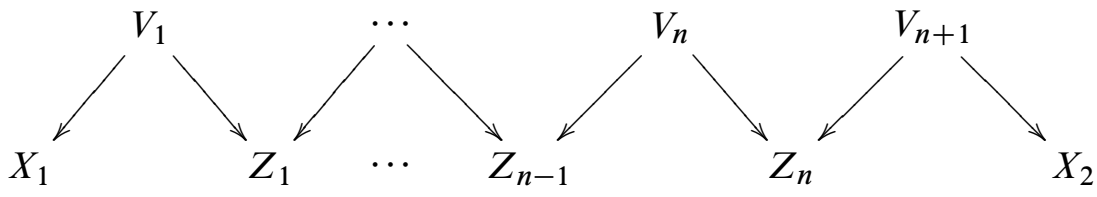

By applying the claim with $V=V_{n}, W=V_{n+1}$, and $X=Z_{n}$, we obtain a hyperbolic $G$-space $A$ which coarsely equivariantly quasi-isometrically embeds into both $V_{n}$ and $V_{n+1}$, and hence into both $Z_{n-1}$ and $X_{2}$. We can thus shorten the sequence (2), unless it is a shortest possible such sequence,

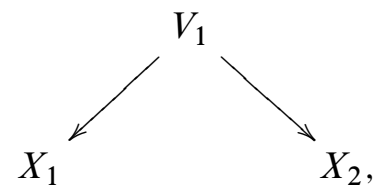

in which case the proposition is verified.

Proof of Claim 3.3 We first construct a $G$-space $A_{1}$ which coarsely equivariantly quasi-isometrically embeds in both $V$ and $W$ and is Gromov hyperbolic but not geodesic. We then show that $A_{1}$ is quasi-isometric to a geodesic $G$-space $A$.

Choose $\delta>0$ so that all of $V, W$, and $X$ are $\delta$-hyperbolic spaces. By hypothesis, we may choose $K \geq 1$ and $C \geq 0$ so that there are maps $\phi: V \rightarrow X$ and $\psi: W \rightarrow Y$ which are $C$-coarsely equivariant $(K, C)$-quasi-isometric embeddings. We choose constants $J_{0}<J_{1}<J_{2}$ : Let $J_{0}=2 B(K, C, \delta)+2 \delta$, where $B(K, C, \delta)$ is the constant of quasi-geodesic stability from Proposition 2.4, let $J_{1}=J_{0}+2 C$, and let $J_{2}=4 J_{1}$. 
Let $A_{0}$ be the subset of $V \times W$ given by $A_{0}=\left\{(v, w) \mid d(\phi(v), \psi(w)) \leq J_{0}\right\}$, and let $A_{1}$ be the smallest $G$-equivariant subset of $V \times W$ containing $A_{0}$. We endow $A_{1}$ with the (pseudo)metric

$$
d\left(\left(v_{1}, w_{1}\right),\left(v_{2}, w_{2}\right)\right)=d\left(\phi\left(v_{1}\right), \phi\left(v_{2}\right)\right)+d\left(\psi\left(v_{2}\right), \psi\left(w_{2}\right)\right) .
$$

Subclaim 3.4 $A_{1}$ is Gromov hyperbolic.

Proof Since $A_{1}$ is not a geodesic space, we must work with the four-point Definition 2.5. We will show that $A_{1}$ is $\left(2 \delta+4 J_{1}\right)$-hyperbolic in the sense of Definition 2.5. Let $\left\{p_{i}=\left(v_{i}, w_{i}\right) \mid i=1, \ldots, 4\right\}$ be four points in $A_{1}$. For each $i$, we write $\underline{v}_{i}$ for $\phi\left(v_{i}\right)$ and $\underline{w}_{i}$ for $\psi\left(w_{i}\right)$. By reordering the points if necessary, we can assume that

$$
d\left(\underline{v}_{1}, \underline{v}_{3}\right)+d\left(\underline{v}_{2}, \underline{v}_{4}\right) \geq d\left(\underline{v}_{1}, \underline{v}_{2}\right)+d\left(\underline{v}_{3}, \underline{v}_{4}\right),
$$

as in Figure 1. There are then two cases.

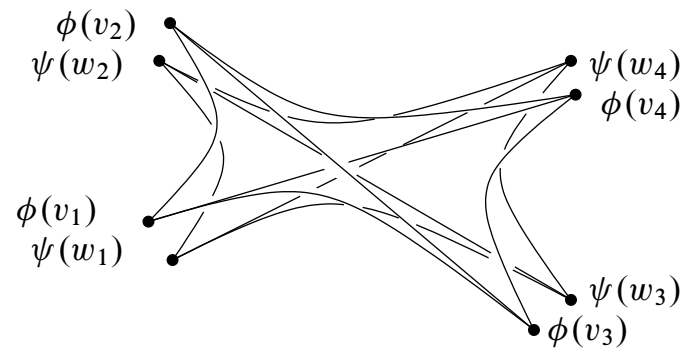

Figure 1: Points in $X$

In case $\quad d\left(\underline{w}_{1}, \underline{w}_{3}\right)+d\left(\underline{w}_{2}, \underline{w}_{4}\right) \geq d\left(\underline{w}_{1}, \underline{w}_{2}\right)+d\left(\underline{w}_{3}, \underline{w}_{4}\right)$,

then $\max \left\{d\left(p_{1}, p_{3}\right)+d\left(p_{2}, p_{4}\right), d\left(p_{1}, p_{2}\right)+d\left(p_{3}, p_{4}\right)\right\}=d\left(p_{1}, p_{2}\right)+d\left(p_{2}, p_{4}\right)$. Applying $\delta$-hyperbolicity in $X$, we obtain

$$
\begin{aligned}
d\left(p_{1}, p_{4}\right)+d\left(p_{3}, p_{2}\right)= & d\left(\underline{v}_{1}, \underline{v}_{4}\right)+d\left(\underline{v}_{2}, \underline{v}_{3}\right)+d\left(\underline{w}_{1}, \underline{w}_{4}\right)+d\left(\underline{w}_{2}, \underline{w}_{3}\right) \\
\leq & d\left(\underline{v}_{1}, \underline{v}_{3}\right)+d\left(\underline{v}_{2}, \underline{v}_{4}\right)+2 \delta+d\left(\underline{w}_{1}, \underline{w}_{3}\right)+d\left(\underline{w}_{2}, \underline{w}_{4}\right)+2 \delta \\
= & d\left(p_{1}, p_{3}\right)+d\left(p_{2}, p_{4}\right)+4 \delta \\
= & \max \left\{d\left(p_{1}, p_{3}\right)+d\left(p_{2}, p_{4}\right), d\left(p_{1}, p_{2}\right)+d\left(p_{3}, p_{4}\right)\right\}+2(2 \delta) \\
\leq & \max \left\{d\left(p_{1}, p_{3}\right)+d\left(p_{2}, p_{4}\right), d\left(p_{1}, p_{2}\right)+d\left(p_{3}, p_{4}\right)\right\} \\
& +2\left(2 \delta+4 J_{1}\right)
\end{aligned}
$$

as required. 
If on the other hand

$$
d\left(\underline{w}_{1}, \underline{w}_{3}\right)+d\left(\underline{w}_{2}, \underline{w}_{4}\right)<d\left(\underline{w}_{1}, \underline{w}_{2}\right)+d\left(\underline{w}_{3}, \underline{w}_{4}\right),
$$

then it is still true $\left(\right.$ since $\left.\left.d\left(\underline{w}_{i}, \underline{v}_{i}\right) \leq J_{1}\right)\right)$ that

$$
\begin{aligned}
d\left(\underline{w}_{1}, \underline{w}_{3}\right)+d\left(\underline{w}_{2}, \underline{w}_{4}\right) & \geq d\left(\underline{v}_{1}, \underline{v}_{3}\right)+d\left(\underline{v}_{2}, \underline{v}_{4}\right)-4 J_{1} \\
& \geq d\left(\underline{v}_{1}, \underline{v}_{2}\right)+d\left(\underline{v}_{3}, \underline{v}_{4}\right)-4 J_{1} \\
& \geq d\left(\underline{w}_{1}, \underline{w}_{2}\right)+d\left(\underline{w}_{3}, \underline{w}_{4}\right)-8 J_{1} .
\end{aligned}
$$

We therefore obtain

$$
\begin{aligned}
d\left(p_{1}, p_{4}\right)+d\left(p_{3}, p_{2}\right)= & d\left(\underline{v}_{1}, \underline{v}_{4}\right)+d\left(\underline{v}_{2}, \underline{v}_{3}\right)+d\left(\underline{w}_{1}, \underline{w}_{4}\right)+d\left(\underline{w}_{2}, \underline{w}_{3}\right) \\
\leq & d\left(\underline{v}_{1}, \underline{v}_{3}\right)+d\left(\underline{v}_{2}, \underline{v}_{4}\right)+2 \delta+d\left(\underline{w}_{1}, \underline{w}_{2}\right)+d\left(\underline{w}_{3}, \underline{w}_{4}\right)+2 \delta \\
\leq & d\left(p_{1}, p_{3}\right)+d\left(p_{2}, p_{4}\right)+2\left(2 \delta+4 J_{1}\right) \\
\leq & \max \left\{d\left(p_{1}, p_{3}\right)+d\left(p_{2}, p_{4}\right), d\left(p_{1}, p_{2}\right)+d\left(p_{3}, p_{4}\right)\right\} \\
& +2\left(2 \delta+4 J_{1}\right),
\end{aligned}
$$

which finishes the proof of the Subclaim.

Though the space $A_{1}$ is hyperbolic, and $G$ quasi-acts on $A_{1}$ via the diagonal action on $V \times W$, the space $A_{1}$ is not geodesic, and $G$ does not act by isometries. Both of these issues can be fixed at once, by replacing $A_{1}$ by an appropriate graph. Specifically, we let $A$ be the graph with vertex set $V(A)=A_{1}$, and with an edge between every pair of vertices $p$ and $q$ so that there exists some $g$ with $d(g p, g q) \leq J_{2}:=4 J_{1}$. Clearly this graph is a geodesic $G$-space.

Let $\iota: A_{1} \rightarrow A$ be the map which takes a point to the corresponding vertex. We claim that $\iota$ is a quasi-isometry, and so $A$ is a Gromov hyperbolic space.

Let $a=\left(v_{a}, w_{a}\right)$ and $b=\left(v_{b}, w_{b}\right)$ be points in $A_{1}$ so that $d(\iota(a), \iota(b))=1$. There is some $g$ with

$$
d(g a, g b)=d\left(\phi\left(g v_{a}\right), \phi\left(g v_{b}\right)\right)+d\left(\psi\left(g v_{a}\right), \psi\left(g v_{b}\right)\right) \leq J_{2} .
$$

Because $\phi$ and $\psi$ are both $C$-coarsely equivariant, $d\left(\phi\left(g v_{a}\right), \phi\left(g v_{b}\right)\right)$ differs by at most $2 C$ from $d\left(\phi\left(v_{a}\right), \phi\left(v_{b}\right)\right)$; similarly, $d\left(\psi\left(g w_{a}\right), \psi\left(g w_{b}\right)\right)$ differs from $d\left(\psi\left(w_{a}\right), \psi\left(w_{b}\right)\right)$ by at most $2 C$. It follows that $d(g a, g b)$ differs by at most $4 C$ from $d(a, b)$, and so $d(a, b) \leq J_{2}+4 C<(3 / 2) J_{2}$. As this is true for every pair of points connected by an edge, we deduce

$$
d(\iota(p), \iota(q)) \geq \frac{2}{3 J_{2}} d(p, q)
$$


for any pair of points $p, q \in A_{1}$.

We now obtain the complementary bound to (3). Suppose $p=\left(v_{1}, w_{1}\right)$ and $q=$ $\left(v_{2}, w_{2}\right)$ are any two points in $A_{1}$. We write $\underline{v}_{1}$ for $\phi\left(v_{1}\right)$ and so on as before. We will show that

$$
d(\iota(p), \iota(q)) \leq \frac{1}{J_{1}} d(p, q)+2
$$

by constructing a path joining $\iota(p)$ to $\iota(q)$ in $A$. The vertices of this path will be points in $A_{0} \subset V \times W$ so that the first coordinate lies on a geodesic between $v_{1}$ and $v_{2}$, while the second lies on a geodesic between $w_{1}$ and $w_{2}$.

If $d(p, q) \leq 2 J_{2}=8 J_{1}$, then (4) is automatically satisfied. We may therefore assume that $d(p, q) \geq 2 J_{2}$. Because $d\left(\underline{v}_{1}, \underline{w}_{1}\right)$ and $d\left(\underline{w}_{1}, \underline{w}_{2}\right)$ are at most $J_{0}$, we have $\min \left\{d\left(\underline{v}_{1}, \underline{v}_{2}\right), d\left(\underline{w}_{1}, \underline{w}_{2}\right)\right\} \geq J_{2}-J_{0}>3 J_{0}$. It follows that $\left(\underline{v}_{2} \cdot \underline{w}_{2}\right)_{\underline{v}_{1}}>\left(\underline{w}_{1} \cdot \underline{w}_{2}\right)_{\underline{v}_{1}}$, as in Figure 2 . In fact, $\left(\underline{v}_{2} \cdot \underline{w}_{2}\right)_{\underline{v}_{1}}-\left(\underline{w}_{1} \cdot \underline{w}_{2}\right)_{\underline{v}_{1}}>J_{0} \geq J_{1} / 2$ (since clearly $B(K, C, \delta) \geq C$ ).
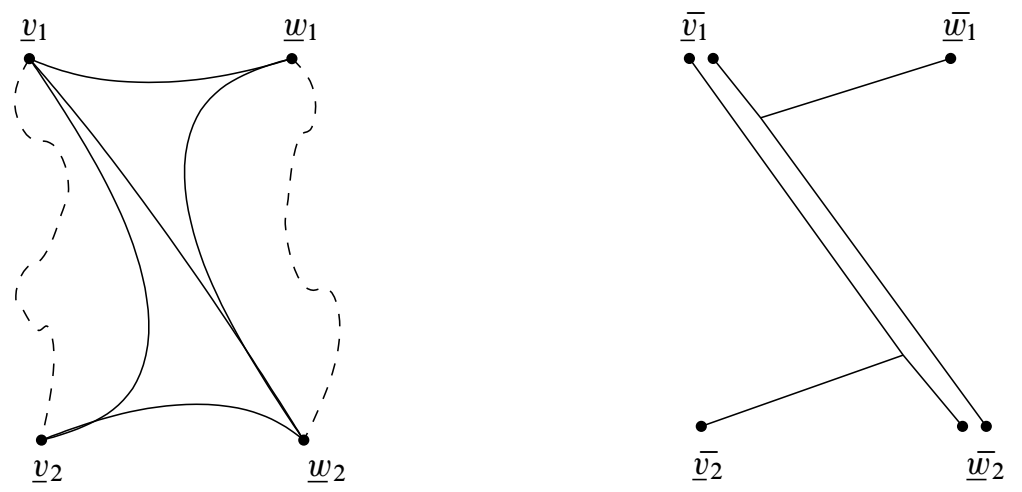

Figure 2: A pair of pairs of points in $X$, together with comparison tripods. The dashed line on the left is the image of a geodesic in $V$; the one on the right is the image of a geodesic in $W$.

We may thus choose real numbers

$$
\left(\underline{w}_{1} \cdot \underline{w}_{2}\right)_{\underline{v}_{1}}=t_{0}<t_{1}<\cdots<t_{k}=\left(\underline{v}_{2} \cdot \underline{w}_{2}\right)_{\underline{v}_{1}}
$$

satisfying $J_{1} / 2<\left|t_{i+1}-t_{i}\right| \leq J_{1}$ for each $i$. Note that $k \leq\left(1 / J_{1}\right) d(p, q)$ for such a choice.

Let $\gamma$ be a unit speed geodesic from $\underline{v}_{1}$ to $\underline{w}_{2}$. For each $i$ between 0 and $k$, there are points $x_{i}^{\prime}$ on a geodesic between $\underline{v}_{1}$ and $\underline{w}_{1}$ and $y_{i}^{\prime}$ on a geodesic between $\underline{w}_{1}$ and $\underline{w}_{2}$ which are distance at most $\delta$ from $\gamma\left(t_{i}\right)$. Since $V$ and $W$ are geodesic spaces, there are 
geodesics $\left[v_{1}, v_{2}\right]$ in $V$ and $\left[w_{1}, w_{2}\right]$ in $W$. The maps $\phi$ and $\psi$ send these geodesics to $(K, C)$-quasi-geodesics in $X$. Applying quasi-geodesic stability (Proposition 2.4), there are points $x_{i} \in\left[v_{1}, v_{2}\right], y_{i} \in\left[w_{1}, w_{2}\right]$ so that $\phi\left(x_{i}\right)$ and $\psi\left(y_{i}\right)$ lie within $B+\delta$ of $\gamma\left(t_{i}\right)$. Since $d\left(\phi\left(x_{i}\right), \psi\left(y_{i}\right)\right) \leq 2 B+2 \delta=J_{0}$, the point $p_{i}=\left(x_{i}, y_{i}\right)$ lies in $A_{0} \subseteq A_{1}$. Moreover, $d\left(p_{i}, p_{i+1}\right) \leq 2\left(2(B+\delta)+J_{1}\right)<J_{2}$, and so $d\left(\iota\left(p_{i}\right), \iota\left(p_{i+1}\right)\right) \leq 1$.

Finally, one notes that

$$
d\left(\underline{v}_{1}, \phi\left(x_{0}\right)\right)+d\left(\underline{w}_{1}, \psi\left(y_{0}\right)\right) \leq J_{0}+2 \delta+2 B=2 J_{0}<J_{2}
$$

and likewise for $d\left(\underline{v}_{1}, \phi\left(x_{0}\right)\right)+d\left(\underline{w}_{1}, \psi\left(y_{0}\right)\right)$. It follows that $d\left(\iota(p), \iota\left(p_{0}\right)\right)$ and $d\left(\iota(q), \iota\left(p_{k}\right)\right)$ are at most one. Thus

$$
d(\iota(p), \iota(q)) \leq k+2 \leq \frac{1}{J_{1}} d(p, q)+2 .
$$

It is obvious that $\iota$ is 1 -almost onto, and so $\iota$ is a quasi-isometry.

It follows that $A$ is a Gromov hyperbolic geodesic $G$-space which coarsely equivariantly, quasi-isometrically embeds into $V$ and $W$.

This completes the proof of Proposition 3.2.

Remark 3.5 We record some observations about this equivalence:

(1) Let $\Gamma$ be a group acting isometrically on $\mathbb{H}^{2}$. This action extends in an obvious way to either $\mathbb{H}^{3}$ or $\mathbb{C} \mathbb{H}^{2}$. Although there is no quasi-isometric embedding either from $\mathbb{H}^{3}$ to $\mathbb{C} \mathbb{H}^{2}$ or vice versa, these actions are equivalent under the equivalence relation.

(2) A Gromov hyperbolic $G$-space has a bounded orbit if and only if it is equivalent to the trivial $G$-space consisting of a single point.

(3) If $X$ and $Y$ are equivalent Gromov hyperbolic $G$-spaces, $x \in X$ and $y \in Y$, then the limit sets $\Lambda(X)=\{e \in \partial X \cap \overline{G x}\}$ and $\Lambda(Y)=\{e \in \partial Y \cap \overline{G y}\}$ are equivariantly homeomorphic.

(4) The equivalence is perfectly well defined in the more general setting of quasiactions on geodesic Gromov hyperbolic spaces. Call a geodesic Gromov hyperbolic space with a $G$-quasi-action a hyperbolic quasi- $G$-space. Every hyperbolic quasi- $G$-space is equivalent to some hyperbolic $G$-space.

Given a group acting on a hyperbolic $G$-space $X$ and some basepoint $x_{0} \in X$, one obtains a metric on $G$ given by the formula

$$
d(g, h)=d\left(x_{0}, g^{-1} h x_{0}\right) .
$$


This metric is obviously determined by its values on $\{1\} \times G$. The compact-open topology on real-valued functions on $G$ thus induces a topology on the set of pointed hyperbolic $G$-spaces. The quotient topology on the space of equivalence classes of Gromov hyperbolic $G$-spaces is not Hausdorff. For example, if $G$ is a surface group, then all of Teichmüller space is identified to a single point, whose closure contains many inequivalent actions of $G$ on $\mathbb{R}$-trees.

\subsection{Combinatorial horoballs}

Combinatorial horoballs of the simplest possible type were defined by Groves and the author [13], and used as building blocks for complexes naturally associated to relatively hyperbolic groups. The point there as here is that these spaces can be used to "hide" an action on a nonhyperbolic space in an action on a hyperbolic space. There is some flexibility as to how this can happen which is deliberately ignored in [13]; here we give a more general construction.

Definition 3.6 Let $X$ be a connected graph, acted on by $G$, and suppose that

$$
\left\{B_{i}: X^{(0)} \rightarrow 2^{X^{(0)}}\right\}_{i \in \mathbb{N}}
$$

is a collection of functions. We will say that $B_{*}$ is admissible if it satisfies the following four axioms:

(1) Connectedness: If $v \in X^{(0)}$, then $B_{1}(v)=\left\{w \in X^{(0)} \mid d_{X}(v, w) \leq 1\right\}$.

(2) Exponential growth: Let $v, w \in X^{(0)}$ and $n \in \mathbb{N}$. If $v \in B_{n}(w)$, then $B_{n}(v) \subset$ $B_{n+1}(w)$.

(3) Symmetry: Let $v, w \in X^{(0)}$ and $n \in \mathbb{N}$. If $v \in B_{n}(w)$, then $w \in B_{n}(v)$.

(4) $G$-equivariance: If $w \in X^{(0)}, n \in \mathbb{N}$, and $g \in G$, then $g B_{n}(w)=B_{n}(g w)$.

Definition 3.7 Let $B_{*}$ be a sequence of functions $\left\{B_{i}: X^{(0)} \rightarrow 2^{X^{(0)}}\right\}_{i \in \mathbb{N}}$. The combinatorial horoball based on $X$ and $B_{*}$, or $\mathcal{H}\left(X, B_{*}\right)$, is the graph defined as follows:

(1) $\mathcal{H}\left(X, B_{*}\right)^{(0)}=X^{(0)} \times \mathbb{N}$.

(2) If $n \in \mathbb{N}$ and $v \in X^{(0)}$, then $(v, n)$ is connected to $(v, n+1)$ by an edge (called a vertical edge).

(3) If $n \in \mathbb{N}$ and $v \in B_{n}(w)$, then $(v, n)$ is connected to $(w, n)$ by an edge (called a horizontal edge).

We leave the following Lemma as an exercise. 
Lemma 3.8 Let $G$ act on the graph $X$. If $B_{*}$ is admissible, then $\mathcal{H}\left(X, B_{*}\right)$ is Gromov hyperbolic, and the action of $G$ on $X$ induces an action of $G$ on $\mathcal{H}\left(X, B_{*}\right)$.

Because of the flexibility of this construction, a group typically admits many inequivalent actions on horoballs.

Definition 3.9 A Gromov hyperbolic $G$-space has an invariant horoball if it is equivalent to a $G$-space of the form $\mathcal{H}\left(X, B_{*}\right)$, for some graph $X$ with a $G$-action, and some family $B_{*}$ which is admissible.

In the next section we give some other characterizations of $G$-spaces with invariant horoballs (Theorem 4.11).

\section{Elementary actions and pseudocharacters}

In this section we show how an elementary action by a finitely generated group $G$ on a hyperbolic space gives rise to a pseudocharacter on $G$ which "picks out" the elements which act hyperbolically. We then see that if no element acts hyperbolically, then the action has an invariant horoball.

\subsection{The pseudocharacter coming from an elementary action}

Recall the following definitions.

Definition 4.1 An action of $G$ on a hyperbolic space $X$ is elementary if it is either equivalent to the trivial action on a point, or if the induced action on $\partial X$ has a global fixed point. ${ }^{1}$

Definition 4.2 A quasi-character (or quasi-morphism) on a group $G$ is a real valued function $q$ on $G$ satisfying

$$
|q(g h)-q(g)-q(h)|<C, \text { for all } g, h \in G .
$$

The defect of a quasi-character is the smallest $C$ so that (5) is satisfied. A pseudocharacter (or homogeneous quasi-morphism) is a quasi-character $p$ which satisfies the additional condition

$$
p\left(g^{n}\right)=n p(g), \text { for all } g \in G, n \in \mathbb{Z} .
$$

\footnotetext{
${ }^{1}$ This definition is slightly more restrictive than the usual one, which allows for a pair of points in $\partial X$ to be preserved.
} 
In this subsection we show how an elementary action on a hyperbolic space gives rise to a pseudocharacter which is nonzero precisely on the elements which act hyperbolically. We begin by studying "quasi-horofunctions" on the space $X$, corresponding to a fixed point at infinity. A quasi-horofunction restricted to an arbitrary orbit will give a quasi-character, which can then be homogenized to give the desired pseudocharacter.

Definition 4.3 (cf Gromov [12, 7.5.D]) Let $\mathbf{x}=\left\{x_{i}\right\}$ be a sequence tending to infinity in the geodesic hyperbolic space $X$. The quasi-horofunction coming from $\mathbf{x}$ is the function $\eta_{\mathbf{x}}: X \rightarrow \mathbb{R}$ given by

$$
\eta_{\mathbf{x}}(a)=\limsup _{n \rightarrow \infty}\left(d\left(a, x_{n}\right)-d\left(x_{0}, x_{n}\right)\right) .
$$

We use the following observation repeatedly:

Observation 4.4 Let $A, B, C$ and $D$ be four points in the $\delta$-hyperbolic space $X$. If $(C \cdot D)_{A}$ and $(C \cdot D)_{B}$ are both larger than $d(A, B)$, then

$$
|(d(B, C)-d(A, C))-(d(B, D)-d(A, D))| \leq 4 \delta .
$$

The Observation 4.4 implies in particular:

Lemma 4.5 If $a \in X$, and $\mathbf{x}=\left\{x_{i}\right\}$ tends to infinity in $X$, then for all $n$ sufficiently large,

$$
\left|\eta_{\mathbf{x}}(a)-\left(d\left(a, x_{n}\right)-d\left(x_{0}, x_{n}\right)\right)\right| \leq 4 \delta
$$

We now can describe the dependence of $\eta_{\mathbf{x}}$ on the sequence $\mathbf{x}$.

Lemma 4.6 Let $\mathbf{x}=\left\{x_{i}\right\}$ and $\mathbf{y}=\left\{y_{i}\right\}$ be two sequences of points in the geodesic $\delta$-hyperbolic space $X$ which tend to the same point in $\partial X$. For any point $a \in X$, we have

$$
\left|\eta_{\mathbf{x}}(a)-\eta_{\mathbf{y}}(a)-\eta_{\mathbf{x}}\left(y_{0}\right)\right| \leq 16 \delta .
$$

Proof Since $\mathbf{x}$ and $\mathbf{y}$ tend to the same point at infinity, we may choose $N$ so that $\left(z, z^{\prime}\right)_{\alpha}>2 \operatorname{diam}\left\{a, x_{0}, y_{0}\right\}$ for every $z, z^{\prime}$ in $\left\{x_{i} \mid i \geq N\right\} \cup\left\{y_{i} \mid i \geq N\right\}$. Using Lemma 4.5 three times, the quantity

$$
\left|\eta_{\mathbf{x}}(a)-\eta_{\mathbf{y}}(a)-\eta_{\mathbf{x}}\left(y_{0}\right)\right|
$$

differs by at most $12 \delta$ from

$$
\left|\left(d\left(a, x_{N}\right)-d\left(a, y_{N}\right)\right)-\left(d\left(y_{0}, x_{N}\right)-d\left(y_{0}, y_{N}\right)\right)\right| .
$$

By Observation 4.4, the quantity (6) is at most $4 \delta$. The Lemma follows. 
Using Lemma 4.6, we deduce that an isometry of $X$ changes $\eta_{\mathbf{x}}(a)$ by approximately the same amount, independent of the $a \in X$ chosen:

Proposition 4.7 Let $X$ be a geodesic $\delta$-hyperbolic space, and suppose that $\mathbf{x}=\left\{x_{i}\right\}$ tends to $e \in \partial X$. Let $a$ be any point in $X$. If $g \in \operatorname{Isom}(X)$ fixes $e$, then $\eta_{\mathbf{x}}(g a)$ differs from $\eta_{\mathbf{x}}(a)+\eta_{\mathbf{x}}\left(g x_{0}\right)$ by at most $16 \delta$.

Proof First note that if $g \mathbf{x}$ is the sequence $\left\{g x_{i}\right\}$, then

$$
\eta_{\mathbf{x}}(a)=\eta_{g_{\mathbf{x}}}(g a) .
$$

But by Lemma 4.6,

$$
\left|\eta_{\mathbf{x}}(g a)-\eta_{g \mathbf{x}}(g a)-\eta_{\mathbf{x}}\left(g x_{0}\right)\right| \leq 16 \delta .
$$

Corollary 4.8 Suppose $X$ is a $\delta$-hyperbolic space, and that $G$ acts on $X$ fixing $e \in \partial X$. Let $\mathbf{x}=\left\{x_{i}\right\}$ be any sequence tending to $e$. The function $q_{\mathbf{x}}: G \rightarrow \mathbb{R}$ defined by $q_{\mathbf{x}}(g)=\eta_{\mathbf{x}}\left(g x_{0}\right)$ is a quasi-character of defect at most $16 \delta$.

Proof Let $g, h \in G$. Using Proposition 4.7,

$$
\begin{aligned}
\left|q_{\mathbf{x}}(g h)-q_{\mathbf{x}}(g)-q_{\mathbf{x}}(h)\right| & =\left|\eta_{\mathbf{x}}\left(g h x_{0}\right)-\eta_{\mathbf{x}}\left(g x_{0}\right)-\eta_{\mathbf{x}}\left(h x_{0}\right)\right| \\
& \leq\left|\eta_{\mathbf{x}}\left(h x_{0}\right)+\eta_{\mathbf{x}}\left(g x_{0}\right)-\eta_{\mathbf{x}}\left(g x_{0}\right)-\eta_{\mathbf{x}}\left(h x_{0}\right)\right|+16 \delta \\
& =16 \delta .
\end{aligned}
$$

Proposition 4.9 Let $X, e, \mathbf{x}$, and $q_{\mathbf{x}}$ be as in the statement of Corollary 4.8, and let the pseudocharacter $p_{\mathbf{x}}: G \rightarrow \mathbb{R}$ be given by

$$
p_{\mathbf{x}}(g)=\lim _{n \rightarrow \infty} \frac{q_{\mathbf{x}}\left(g^{n}\right)}{n} .
$$

Then $p_{\mathbf{x}}(g) \neq 0$ if and only if $g$ acts hyperbolically on $X$.

Proof First, we suppose that $p_{\mathbf{x}}(g) \neq 0$. Without loss of generality we assume that $p_{\mathbf{x}}(g)>0$. Since

$$
p_{\mathbf{x}}(g)=\lim _{n \rightarrow \infty} \frac{\eta_{\mathbf{x}}\left(g^{n} x_{0}\right)}{n}>0,
$$

there exists some $N$ so that $\eta_{\mathbf{x}}\left(g^{n} x_{0}\right)>(1 / 2) p_{\mathbf{x}}(g) n$ for all $n \geq N$. 
Let $a$ and $b$ be integers. Choosing some sufficiently large $M$ and applying the triangle inequality and Lemma 4.5, we obtain a lower bound for $d\left(g^{a} x_{0}, g^{b} x_{0}\right)$ :

$$
\begin{aligned}
d\left(g^{a} x_{0}, g^{b} x_{0}\right) & =d\left(g^{N} x_{0}, g^{N+|b-a|} x_{0}\right) \\
& \geq d\left(g^{N+|b-a|} x_{0}, x_{M}\right)-d\left(g^{N} x_{0}, x_{M}\right) \\
& \geq \eta_{\mathbf{x}}\left(g^{N+|b-a|}\right)-\eta_{\mathbf{x}}\left(g^{N}\right)-8 \delta \\
& \geq \frac{1}{2} p_{\mathbf{x}}(g)(N+|b-a|)-\eta_{\mathbf{x}}\left(g^{N}\right)-8 \delta \\
& =\frac{1}{2} p_{\mathbf{x}}(g)|b-a|-\left(\eta_{\mathbf{x}} g^{N}+8 \delta-\frac{1}{2} p_{\mathbf{x}}(g) N\right) .
\end{aligned}
$$

On the other hand, $d\left(g^{a} x_{0}, g^{b} x_{0}\right) \leq|b-a| d\left(x_{0}, g x_{0}\right)$, so the map $n \mapsto g^{n}\left(x_{0}\right)$ is a quasi-isometric embedding, and $g$ acts hyperbolically.

Conversely, suppose that $g$ acts hyperbolically. It follows that there is some $\epsilon>0$ so that

$$
d\left(g^{n} x_{0}, x_{0}\right)>\epsilon n
$$

for all $n$. By replacing $g$ with $g^{-1}$, we may suppose that $\left\{g^{i} x_{0}\right\}$ tends to a point in $\partial X \backslash\{e\}$ as $i \rightarrow \infty$. Thus there is some $R$ so that

$$
\left(g^{n} x_{0} \cdot x_{i}\right)_{x_{0}}<R
$$

for all positive $n$ and $i$. Lemma 4.5 implies that for sufficiently large $m$,

$$
\begin{aligned}
\eta_{\mathbf{x}}\left(g^{n} x_{0}\right) & \geq d\left(g^{n} x_{0}, x_{m}\right)-d\left(x_{0}, x_{m}\right)-4 \delta \\
& =d\left(g^{n} x_{0}, x_{0}\right)-2\left(g^{n} x_{0} \cdot x_{n}\right)_{x_{0}}-4 \delta \\
& \geq \epsilon n-(2 R+4 \delta) .
\end{aligned}
$$

Since $q_{\mathbf{x}}\left(g^{n}\right) \geq \epsilon n-(2 R+4 \delta)$ for all $n>0$, we must have $p(g) \geq \epsilon>0$.

Remark 4.10 Proposition 4.9 was proved in [18, Proposition 3.9] for the case of quasi-trees. The proof here is somewhat more efficient even in this case.

\subsection{Characterization of $G$-spaces with invariant horoballs}

Theorem 4.11 Let $X$ be a Gromov hyperbolic $G$-space. The following are equivalent:

(1) $X$ has an invariant horoball.

(2) $X$ is elementary, and no element acts hyperbolically.

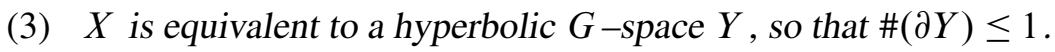


Proof That (1) implies (3) is trivial.

We next assume (3) and show (2). If (2) holds for $Y$, it holds for $X$, so we may suppose that $X=Y$. If $\#(\partial X)=1$ or $G$ has a bounded orbit in $X$, then clearly $X$ is elementary. The only case remaining is that $\partial X$ is empty, but $G x$ is unbounded for some $x \in X$. We show that this case does not occur. Chose a sequence $\left\{g_{i}\right\}$ in $G$ so that $\lim _{i \rightarrow \infty} d\left(g_{i} x, x\right)=\infty$. Since $\partial X$ is empty, $\liminf _{i, j \rightarrow \infty}\left(g_{i} x, g_{j} x\right)_{x}<\infty$. It follows that there are elements $g_{m}, g_{n}$ so that $d\left(g_{m} x, x\right)$ and $d\left(g_{n} x, x\right)$ are much larger than $\left(g_{m} x, h_{n} x\right)_{x}$. It can then be shown (see, for example, [8, Chapitre 9, Lemme 2.3]) that $g_{m} g_{n}$ is hyperbolic. It follows that $\partial X$ contains at least two points (the fixed points of $g_{m} g_{n}$ ), contrary to assumption.

It remains to show that (2) implies (1). Let $X$ be a hyperbolic $G$-space so that the action of $G$ is elementary. If $X$ is equivalent to a point (ie if $G x$ is bounded for $x \in X$ ), then $X$ is also equivalent to a ray, which is the combinatorial horoball based on a point. We therefore may assume that $G x$ is unbounded for any $x \in X$. We will construct a combinatorial horoball which coarsely equivariantly quasi-isometrically embeds in $X$. Let $\delta>0$ be some number so that $X$ is $\delta$-hyperbolic. Let $e, \mathbf{x}, q_{\mathbf{x}}$ and $p_{\mathbf{x}}$ be as in the statements of Corollary 4.8 and Proposition 4.9.

To build the combinatorial horoball, we first must start with a graph $Y$ on which $G$ acts. Choose a finite generating set $S$ for $G$, and let $C_{0}=\operatorname{diam}\left(S x_{0}\right)$. Let $V(Y)=G$, and connect $g$ to $h$ in $Y$ if $d\left(g x_{0}, h x_{0}\right) \leq C_{0}$. It is clear that $G$ acts on $Y$; in fact, $Y$ is a certain Cayley graph for $G$. We next define the functions $B_{n}: V(Y) \rightarrow 2^{V(Y)}$. Let $C_{1}=2 C_{0}+20 \delta$, and let

$$
B_{n}(g)=\left\{h \in G \mid d\left(h x_{0}, g x_{0}\right) \leq(2 n+1) C_{1}\right\} .
$$

Claim 4.12 The sequence of functions $B_{*}$ in (7) is admissible in the sense of Definition 3.6.

Proof The only axiom which is not obvious is (2). We must show that if $a$ and $b$ are in $B_{n}(v)$, then $a \in B_{n+1}(b)$ (or equivalently $b \in B_{n+1}(a)$ ). Put another way, we must show that if $d\left(a x_{0}, v x_{0}\right)$ and $d\left(b x_{0}, v x_{0}\right)$ are bounded above by $(2 n+1) C_{1}$ then $d\left(a x_{0}, b x_{0}\right) \leq(2 n+3) C_{1}$.

Because no element of $G$ acts hyperbolically, the pseudocharacter $p_{\mathbf{x}}$ is identically zero. An easy argument shows that $\left|q_{\mathbf{x}}(g)\right| \leq 16 \delta$ for all $g \in G$. Using Lemma 4.5, we can choose some large $n$ so that

$$
\left|\eta_{\mathbf{x}}(z)-\left(d\left(z, x_{n}\right)-d\left(x_{0}, x_{n}\right)\right)\right| \leq 4 \delta
$$


for $z \in\left\{a x_{0}, b x_{0}, v x_{0}\right\}$. It follows that

$$
\left|d\left(z_{1}, x_{m}\right)-d\left(z_{2}, x_{m}\right)\right| \leq 16 \delta+4 \delta \leq C_{1}
$$

for $z_{1}, z_{2} \in\left\{a x_{0}, b x_{0}, v x_{0}\right\}$. The assertion to be proved is symmetric in $a$ and $b$, so we may assume that $\left(x_{m} \cdot b\right)_{v} \leq\left(x_{m} \cdot a\right)_{v}$. We deduce:

$$
\begin{aligned}
d(a, b) & \leq\left(v \cdot x_{m}\right)_{a}+\left[\left(a \cdot x_{m}\right)_{v}-\left(b \cdot x_{m}\right)_{v}\right]+\left(v \cdot x_{m}\right)_{b}+2 \delta \\
& =d(v, a)+\left(d\left(b, x_{m}\right)-d\left(v, x_{m}\right)\right)+2 \delta \\
& \leq(2 n+1) C_{1}+C_{1}+2 \delta \leq(2 n+3) C_{1} .
\end{aligned}
$$

The first line follows from studying the comparison tripods for the triangles $\Delta\left(a, v, x_{m}\right)$ and $\Delta\left(b, v, x_{m}\right)$; the last follows from (8).

Since $B_{*}$ is admissible, the combinatorial horoball $H=\mathcal{H}_{B_{*}}(Y)$ is a hyperbolic $G-$ space. It remains to construct a coarsely equivariant quasi-isometric embedding from $H$ to $X$. It suffices to define this map on the vertices of $H$. For each $g \in G=V(Y)$ and each $n \in \mathbb{N}$, choose some $i(g, n)$ so that $\left(x_{k} \cdot x_{l}\right)_{g x_{0}} \geq 2 n C_{1}$ for all $k, l \geq i(g, n)$. Choose also some unit speed geodesic $\gamma_{g, n}$ starting at $g x_{0}$ and ending at $x_{i(g, n)}$. Any vertex of $H$ is a pair $(g, n)$ where $n \in \mathbb{N}$ and $g \in G$. Define a map $\phi: V(H) \rightarrow X$ by

$$
\phi(g, n)=\gamma_{g, n}\left(n C_{1}\right) .
$$

A number of choices were made in the definition of $\phi$ (namely, the sequence $\mathbf{x}$, the numbers $i(g, n)$, and the geodesics $\left.\gamma_{g, n}\right)$. However, so long as $x_{0}$ is unchanged, different choices lead to a function which differs by at most $\delta$ from $\phi$. In particular, we could replace $\mathbf{x}$ by $\mathbf{x}^{\prime}=\left\{x_{i}^{\prime}\right\}$, where $x_{0}^{\prime}=x_{0}$ and $x_{i}^{\prime}=h x_{i}$ for some fixed $h$ and for all $i \geq 1$. It follows that the distance between $\phi(h g, n)$ and $h \phi(g, n)$ is at most $\delta$ for any $h, g \in G$ and $n \in \mathbb{N}$, and so the map $\phi$ is coarsely equivariant.

It remains to show that $\phi$ is a quasi-isometric embedding.

Note that if $v$ and $w$ are two vertices in $H$ connected by a vertical path, then $C_{1} d_{H}(v, w)-\delta \leq d(\phi(v), \phi(w)) \leq C_{1} d_{H}(v, w)+\delta$, where $d_{H}$ is the distance in $H$.

We therefore assume that $v=(a, n)$ and $w=(b, m)$, where $a \neq b$. There is a unique $k$ so that $(2 k-1) C_{1}<d(a, b) \leq(2 k+1) C_{1}$. If $\max \{m, n\} \geq k$, then $d(v, w)=|m-n|+1$; otherwise $d(v, w)=2 k-(m+n)+1 / 2 \pm 1 / 2$. Let $I \geq$ $\max \{i(a, n), i(b, m)\}$, and observe that the points $\phi(v)$ and $\phi(w)$ lie within $\delta$ of geodesics joining $a x_{0}$ to $x_{I}$ and $b x_{0}$ to $x_{I}$, respectively (see Figure 3 ). Note that $\left(x_{I} \cdot a x_{0}\right)_{b x_{0}}$ and $\left(x_{I} \cdot b x_{0}\right)_{a x_{0}}$ differ from $1 / 2 d\left(a x_{0}, b x_{0}\right)$ by at most $C_{1} / 2$. There are a couple of cases to consider. 

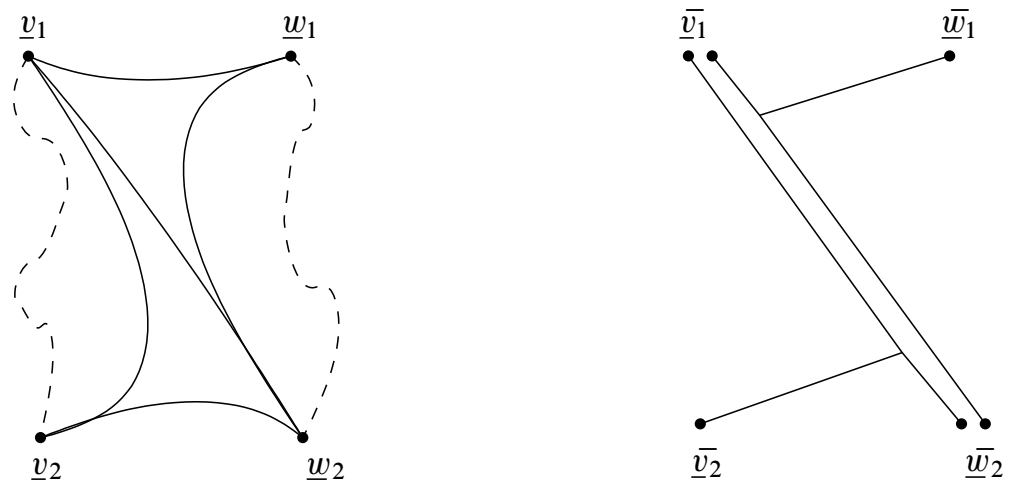

Figure 3: A pair of points in the image of $\phi$

First, assume that one or both of $n$ and $m$ is at least $k$. Without loss of generality assume that $n \geq k$. Since $d\left(a x_{0}, b x_{0}\right) \leq(2 k+1) C_{1}$, we have $\left(x_{I}, b x_{0}\right)_{a} \leq k C_{1}+C_{1}$. Since $d\left(\phi(v), a x_{0}\right) \geq k C_{1}$, it follows that $\phi(v)$ is at most $C_{1}+\delta$ from the geodesic joining $b x_{0}$ to $x_{I}$. Accounting for the possible difference between $\left(x_{I} \cdot a x_{0}\right)_{b x_{0}}$ and $\left(x_{I} \cdot b x_{0}\right)_{a x_{0}}$, we deduce that the distance between $\phi(v)$ and $\phi(w)$ differs from $|n-m| C_{1}=(d(v, w)+1 / 2 \pm 1 / 2) C_{1}$ by at most $2 C_{1}+\delta$. It follows that in case one of $n$ or $m$ is at least $k$, we have

$$
C_{1} d(v, w)-2 C_{1}+\delta \leq d(\phi(v), \phi(w)) \leq C_{1} d(v, w)+3 C_{1}+\delta
$$

In case both $n$ and $m$ are strictly less than $k$, we may argue as follows. Since $\left(a x_{0} \cdot x_{I}\right)_{b x_{0}}$ and $\left(b x_{0} \cdot x_{I}\right)_{a x_{0}}$ are both at least $1 / 2\left(d\left(a x_{0}, b x_{0}\right)-C_{1}\right) \geq(k-1) C_{1} \geq$ $\max \{m, n\} C_{1}$, it follows that both $\phi(v)$ and $\phi(w)$ are within $2 \delta$ of the geodesic joining $a x_{0}$ to $b x_{0}$. From this it follows that $d(\phi(v), \phi(w))$ differs from $d\left(a x_{0}, b x_{0}\right)-$ $(n+m) C_{1}$ by at most $4 \delta$. But since $d\left(a x_{0}, b x_{0}\right)$ differs by at most $C_{1}$ from $k C_{1}$, we deduce that

$$
(2 k-(m+n)) C_{1}-\left(C_{1}+4 \delta\right) \leq d(\phi(v), \phi(w)) \leq(2 k-(m+n)) C_{1}+\left(C_{1}+4 \delta\right),
$$

from which it immediately follows that

$$
C_{1} d(v, w)-\left(2 C_{1}+4 \delta\right) \leq d(\phi(v), \phi(w)) \leq C_{1} d(v, w)+\left(2 C_{1}+4 \delta\right) .
$$

In particular, $\phi$ is a $\left(C_{1}, 2 C_{1}+4 \delta\right)$-quasi-isometric embedding from the combinatorial horoball $H$ into $X$, and the theorem is established. 


\section{Rigidity in rank $\geq 2$}

The purpose of this section is to establish Theorem 1.3, but we will begin with some lemmas which hold in a slightly broader context. Changing notation, we suppose that $G$ is a simple Chevalley-Demazure group scheme of rank at least 2, that $\Phi$ is a root system for $G$, and that $R$ is some commutative unital ring containing $\mathbb{Z}$.

Lemma 5.1 If $\alpha$ and $\beta \in \Phi$, then there is a $\Phi^{\prime}=\operatorname{Span}\left(\Phi^{\prime}\right) \cap \Phi \subseteq \Phi$ containing $\alpha$ and $\beta$ so that $\Phi^{\prime}$ is isomorphic to $A_{1} \times A_{1}, A_{2}, B_{2}$ or $G_{2}$. If $\Phi^{\prime} \cong A_{1} \times A_{1}$, then $\alpha \neq-\beta$.

Proof If $\alpha$ and $\beta$ are linearly independent, then $\Phi^{\prime}=\operatorname{Span}(\{\alpha, \beta\}) \cap \Phi$ is a root system of rank two, and we simply recall that such a root system is always isomorphic to one of those listed.

If $\beta=-\alpha$, we assert that there must be some $\gamma \in \Phi$ so that $\operatorname{Span}(\{\alpha, \gamma\}) \cap \Phi$ is not equal to $A_{1} \times A_{1}$. Suppose that there is no such $\gamma$. Then either $\Phi=\bar{\Phi} \times\langle\alpha\rangle$, or $\Phi=\langle\alpha\rangle$. Because $G$ is simple $\Phi$ cannot split as a product; because $G$ has rank at least two, $\Phi \neq A_{1}$.

For the following two lemmas, we refer to Carter [6]. Although the proofs there are done assuming that $R$ is a field, this assumption is unnecessary; see also Stein [26] or Steinberg [27].

Lemma 5.2 (Steinberg commutator relations [6, Theorem 5.2.2]) If $\alpha, \beta \in \Phi$ and $t$, $u \in R$, then

$$
\left[x_{\alpha}(t), x_{\beta}(u)\right]=\prod_{\substack{i, j>0 \\ i \alpha+j \beta \in \Phi}} x_{i \alpha+j \beta}\left(N_{\alpha, \beta, i, j} t_{i} u_{j}\right),
$$

where the $N_{\alpha, \beta, i, j} \in \mathbb{Z}$ are integers which depend only on the order in which the product is taken.

Lemma 5.3 [6, Lemma 7.2.1] If $\alpha \in \Phi, w$ is an element of the Weyl group of $\Phi$, and $t \in R$, then $x_{w(\alpha)}(t)$ is conjugate either to $x_{\alpha}(t)$ or $x_{\alpha}(-t)$.

Lemma 5.4 Let $X$ be a hyperbolic $G(R)$-space. If $g=x_{\alpha}(t)$ for some $\alpha \in \Phi$ and $t \in R$, then $g$ does not act hyperbolically on $X$.

Proof By Lemma 5.1, there is a subset $\Phi^{\prime}$ of $\Phi$ containing $\alpha$ which is either isomorphic to $A_{2}, B_{2}$ or $G_{2}$. In each case, we may apply Lemma 5.2 some number of times to show that $g$ is distorted in $G$; the details of this are left to the reader. By Lemma 2.12, $g$ cannot act hyperbolically on $X$. 
Proposition 5.5 Let $X$ be a hyperbolic $G(R)$-space, let $r, s \in \Phi$, and let $\rho_{1}, \rho_{2} \in R$. Suppose that $p=x_{r}\left(\rho_{1}\right)$ acts parabolically, fixing some $e \in \partial X$. Then $g e=e$ for any other root element $g=x_{s}\left(\rho_{2}\right)$.

Proof If $r=s$ or if $\langle r, s\rangle=A_{1} \times A_{1}$, then $p$ and $g$ commute. By Lemma 2.10, $g(e)=e$, and we are done.

Otherwise $r$ and $s$ are contained in a two-dimensional root system $\Phi^{\prime} \subset \Phi$ which is isomorphic to $A_{2}, B_{2}$ or $G_{2}$, by Lemma 5.1.

Each case requires a separate argument.

Case $1 \Phi^{\prime} \cong A_{2}$.

$A_{2}=\left\{\lambda_{i} \mid i \in \mathbb{Z}_{6}\right\}$ contains six roots, arranged hexagonally; the angle between $\lambda_{i}$ and $\lambda_{j}$ is $|i-j| / 3 \pi$. Suppose $r=\lambda_{i}$ and $s=\lambda_{j}$. By Lemma 5.3, $h=x_{s}\left(\rho_{1}\right)$ is conjugate to either $p$ or $p^{-1}$, so $h$ is a parabolic, fixing some point $f \in \partial X$. In case $|i-j|=1$, then Lemma 5.2 implies that $h$ and $p$ commute, and so $f=e$ by Lemma 2.10. In case $|i-j|>1$, one argues by induction on $|i-j|$ to the same conclusion: $f=e$.

Since $g$ commutes with $h$, we must have $g e=e$, again by Lemma 2.10.

Case $2 \Phi^{\prime} \cong B_{2}$.

Let $\alpha$ be a short root, and $\beta$ a long root, so that $\alpha$ and $\beta$ span $\Phi^{\prime} \subset \Phi$, as in Figure 4 .
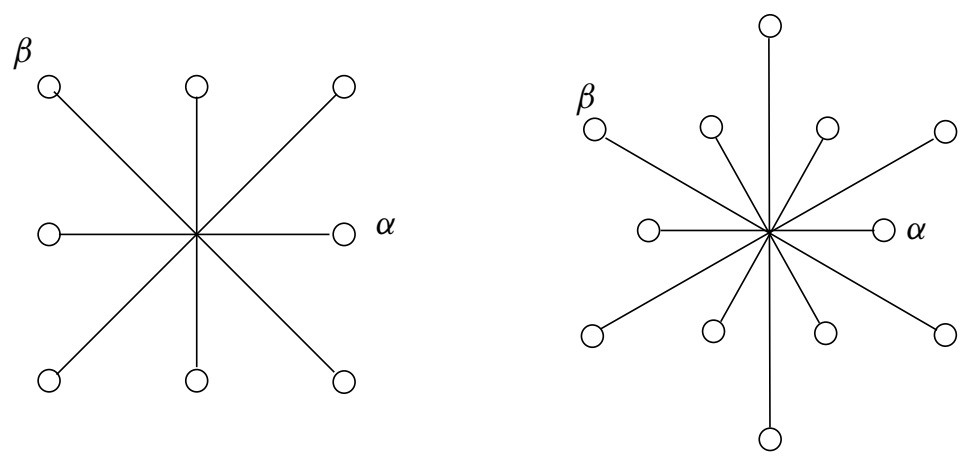

Figure 4: $B_{2}$ and $G_{2}$

There are two subcases, depending on whether $r$ is a short or long root. 
Case 2.1 The parabolic $p=x_{r}\left(\rho_{1}\right)$, where $r$ is a short root of $\Phi^{\prime}$.

Without loss of generality, we may assume that $r=\alpha$. If $s \in\{\alpha, 2 \alpha+\beta,-\beta\}$, then $g=x_{s}\left(\rho_{2}\right)$ commutes with $p$, by Lemma 5.2. Thus by Lemma $2.10 g e=e$.

Suppose next that $s= \pm(\alpha+\beta)$. Then Lemma 5.2 implies

$$
p g p^{-1} g^{-1}=x_{r+s}\left(N \rho_{1} \rho_{2}\right),
$$

where $N=N_{r, s, 1,1}$ is an integer. Since $r+s \in\{2 \alpha+\beta,-\beta\}$, we already know that $h:=x_{r+s}\left(N \rho_{1} \rho_{2}\right)$ fixes $e$. We rearrange (9) to give

$$
h^{-1} p=g p g^{-1} \text {. }
$$

Since $p$ and $h$ both fix $e$, so must $g p g^{-1}$. The element $g p g^{-1}$ also fixes $g(e)$, since $p$ fixes $e$. Since $p$ is parabolic, it can only fix one point in $\partial X$, and so $g(e)=e$.

If $s \in\{\beta,-\alpha\}$, let $r^{\prime}=\alpha+\beta$; if $s=-2 \alpha-\beta$ let $r^{\prime}=-\alpha-\beta$. In any case there is an element of the Weyl group of $\Phi$ taking $r$ to $r^{\prime}$; by Lemma 5.3, there is a $p^{\prime}=x_{r^{\prime}}\left( \pm \rho_{1}\right)$ which is conjugate to $p$ in $E(\Phi, R)$. Since $p^{\prime}$ is conjugate to $p$, it is parabolic; by the argument of the previous paragraph, $p^{\prime}$ has the same fixed point as $p$. If $s \in\{\beta,-2 \alpha-\beta\}$, then $g$ commutes with $p^{\prime}$, and so $g(e)=e$ by Lemma 2.10. Finally, if $s=-\alpha$, then we may apply the argument of the previous paragraph again (with $p^{\prime}$ and $r^{\prime}$ in place of $p$ and $r$ ), to deduce that $g(e)=e$.

Case 2.2 The parabolic $p=x_{r}\left(\rho_{1}\right)$, where $r$ is a long root of $\Phi^{\prime}$.

In this case, we may assume for instance that $r=2 \alpha+\beta$. If $s \in\{2 \alpha+\beta, \alpha+$ $\beta, \alpha, \beta,-\beta$, then Lemma 5.2 implies that $g=x_{s}\left(\rho_{2}\right)$ commutes with $p$, and so $g(e)=e$ by Lemma 2.10 .

Suppose then that $s \in\{-\alpha,-2 \alpha-\beta,-\alpha-\beta\}$. If $s \in\{-\alpha,-2 \alpha-\beta\}$, let $r^{\prime}=\beta$; if $s=-\alpha-\beta$, then let $r^{\prime}=-\beta$. In either case, there is an element of the Weyl group taking $r$ to $r^{\prime}$, and so there is a parabolic $p^{\prime}=x_{r^{\prime}}\left( \pm \rho_{1}\right)$ conjugate to $p$ by Lemma 5.3. By the previous paragraph, $p^{\prime}$ has the same fixed point as $p$ does. Applying the previous paragraph with $p^{\prime}$ and $r^{\prime}$ in the place of $p$ and $r$ implies that $g(e)=e$ for $g=x_{s}\left(\rho_{2}\right)$. This completes the proof of Case 2.

Case $3 \Phi^{\prime} \cong G_{2}$.

Let $\alpha$ be a short root, and $\beta$ a long root, so that $\alpha$ and $\beta$ span $\Phi^{\prime} \subset \Phi$, as in the right half of Figure 4.

Again there are two subcases, depending on whether $r$ is a short or long root. 
Case 3.1 The parabolic $p=x_{r}\left(\rho_{1}\right)$, where $r$ is a long root of $\Phi^{\prime}$.

If $s \in\{-\beta, 3 \alpha+\beta, \pm(3 \alpha+2 \beta)\}$, then Lemma 5.2 implies that $g=x_{s}\left(\rho_{2}\right)$ commutes with $p$, and so $g(e)=e$ by Lemma 2.10 .

Suppose that $s \in\{2 \alpha+\beta,-\alpha-\beta\}$. Lemma 5.2 implies that

$$
p g p^{-1} g^{-1}=x_{r+s}\left(N \rho_{1} \rho_{2}\right)=: h,
$$

for some integer $N$. Exactly as in Case $2, h$ commutes with $p$, and so $h(e)=e$. Thus $e=h^{-1} p(e)=g p g^{-1}(e)$ and the parabolic $g p g^{-1}$ fixes $e$. Again since $g p g^{-1}$ also fixes $g(e)$, we must have $g(e)=e$.

Using Lemma 5.3 repeatedly we discover that for every short root $r^{\prime}$ there is a parabolic element $p^{\prime}=x_{r^{\prime}}\left( \pm \rho_{1}\right)$ with $p^{\prime}(e)=e$. Since $g$ must commute with some such element, $g(e)=e$ as well.

Case 3.2 The parabolic $p=x_{r}\left(\rho_{1}\right)$, where $r$ is a long root of $\Phi^{\prime}$.

Without loss of generality, we may assume that $r=3 \alpha+2 \beta$.

If the inner product of $s$ with $r$ is nonnegative, then $s \in\{ \pm \alpha, \beta, \alpha+\beta, \alpha+2 \beta$, $\alpha+3 \beta, 3 \alpha+2 \beta\}$, and $g$ commutes with $p$ by Lemma 5.2, and so $g(e)=e$.

Otherwise, a (possibly repeated) application of Lemma 5.3 implies that $g$ commutes with a parabolic $p^{\prime}=x_{r^{\prime}}\left( \pm \rho_{1}\right)$ for some long root $r$ of $\Phi^{\prime}$, and with $p^{\prime}(e)=e$. This again implies via Lemma 2.10 that $g(e)=e$. This completes the proof in Case 3 .

We are now ready to give the proof of Theorem 1.3.

Proof A result of Tavgen' [29] shows that $G(\mathcal{O})$ is boundedly generated by its root subgroups. The ring of integers $\mathcal{O}$ is finitely generated as an Abelian group; choose generators $\mu_{1}, \ldots, \mu_{k}$. It follows from Tavgen's result that the set

$$
S=\left\{x_{\alpha}\left(\mu_{i}\right) \mid \alpha \in \Phi, 1 \leq i \leq k\right\}
$$

boundedly generates $G(\mathcal{O})$.

Each of these generators acts hyperbolically, elliptically or parabolically on $X$. By Lemma 5.4, none can act hyperbolically. If all of the root elements act elliptically, then it follows from bounded generation that the orbit of a point under the action of $G$ must be bounded.

We therefore may assume that some $x_{\alpha}\left(\mu_{i}\right)$ acts parabolically on $X$, fixing a single point $e \in \partial X$. It follows from Proposition 5.5 that all the root subgroups will fix this point $e$, and so $G(\mathcal{O})$ fixes $e$. 
By Proposition 4.9, the pseudocharacter $p_{\mathrm{x}}: G(\mathcal{O}) \rightarrow \mathbb{R}$ determined by a sequence $\mathbf{x}=\left\{x_{i}\right\}$ tending to $e$ is nonzero exactly on the hyperbolic elements. Thus $p_{\mathbf{x}}(g)=0$ whenever $g$ lies in a root subgroup.

An elementary argument (see for example Kotschick [16, Proposition 5]) shows that a pseudocharacter $p$ on a boundedly generated group is determined by its values on the bounded generators; thus $p \equiv 0$ on $G(\mathcal{O})$. It follows that no element of $G(\mathcal{O})$ acts hyperbolically on $X$. By Theorem 4.11, the $G(\mathcal{O})$-space $X$ has an invariant horoball.

\section{Remarks on rank one}

One can also ask what actions rank one Chevalley groups have on hyperbolic spaces. If $\mathcal{O}$ is a number ring with finitely many units, then $S L(2, \mathcal{O})$ is a lattice either in $S L(2, \mathbb{R})$ or $S L(2, \mathbb{C})$. In particular, it has a proper nonelementary action on $\mathbb{H}^{2}$ or $\mathbb{H}^{3}$. Moreover, such a group admits uncountably many distinct pseudocharacters (AKA homogeneous quasi-(homo)morphisms) up to scale [9; 2]. Each such "projective pseudocharacter" gives rise to a quasi-action on $\mathbb{R}$; no two such are equivalent. Moreover, these often give rise to quasi-actions on more complicated trees [17]. The groups $S L(2, \mathcal{O})$ where $\mathcal{O}$ has infinitely many units are more rigid. In this section we apply the main result of [18] to the special case of actions on quasi-trees (defined below), and speculate on the general situation.

Recall that a group $G$ is said to have Property (FA) if every action by $G$ on a simplicial tree $T$ has a fixed point.

Definition 6.1 A quasi-tree is a graph which is quasi-isometric to a tree.

Definition 6.2 A group $G$ has property (QFA) if every action by $G$ on a quasi-tree $X$ has a bounded orbit.

Remark 6.3 This is differently worded than the definition in [18], but easily seen to be equivalent. Note that (QFA) implies (FA), but not vice versa.

As quasi-trees are in particular Gromov hyperbolic spaces which admit no parabolic isometries (see Section 3.2 of [18]), Theorem 1.3 implies that higher rank Chevalley groups over number rings have property (QFA).

We recall a definition and a theorem from [18, Section 4]. 
Definition 6.4 Let $G$ be a group, and let $g$ be an element of $G$. We will say $g$ is a stubborn element of $G$ if for all $H<G$ with $[G: H] \leq 2$, there exists some integer $k_{H}>0$ so that $g^{k_{H}} \in[H, H]$.

Theorem 6.5 [18, Theorem 4.4] Let $G$ be a group which is boundedly generated by elements $g_{1}, \ldots, g_{n}$, so that for each $i, g_{i}$ is a stubborn element of $B_{i}$ for some amenable $B_{i}<G$. Then $G$ has Property (QFA).

Note that the above theorem was misstated slightly in [18]; the word "amenable" was inadvertently omitted.

Here's an easy lemma:

Lemma 6.6 Let $H<G$ be a subgroup of finite index. If $H$ has Property (QFA), then so does $G$.

Our methods in the higher rank case use heavily the bounded generation of $G(\mathcal{O})$ established by Tavgen' in [29] for Chevalley groups over rings of integers of algebraic number fields. There is an analogous result of Carter, Keller, and Paige in rank 1, at least for $S L(2, \cdot)$ and certain number fields:

Theorem 6.7 [22] For any integer $d>1$ there is an $r=r(d)$ so that the following is true. Let $K$ be a number field of degree $d$ over $\mathbb{Q}$, and let $\mathcal{O}$ be the ring of integers of $K$. If the $\mathcal{O}$ has infinitely many units, then:

(1) every element of $E(2, \mathcal{O})$ is a product of at most $r$ elementary matrices, and

(2) the index of $E(2, \mathcal{O})$ in $S L(2, \mathcal{O})$ is at most $r$.

In the above statement, $E(2, \mathcal{O})$ is the subgroup of $S L(2, \mathcal{O})$ generated by the root subgroups (the strictly upper triangular and strictly lower triangular matrices). The following statement implies Theorem 1.4:

Theorem 6.8 If $\mathcal{O}$ is the ring of integers of an algebraic number field and $\mathcal{O}$ has infinitely many units, then $S L(2, \mathcal{O})$ has Property (QFA).

Proof By Lemma 6.6 it suffices to show that $E(2, \mathcal{O})$ has property (QFA). If $\Lambda=\left\{\lambda_{1}, \ldots, \lambda_{n}\right\}$ is an integral basis for the number field $\mathcal{O}$, then Theorem 6.7 implies that $E(2, \mathcal{O})$ is boundedly generated by the $2 n$ elements

$$
\left(\begin{array}{cc}
1 & \lambda_{i} \\
0 & 1
\end{array}\right) \text { and }\left(\begin{array}{cc}
1 & 0 \\
\lambda_{i} & 1
\end{array}\right)
$$


Claim 6.9 For each $i \in\{1, \ldots, n\}$,

$$
\left(\begin{array}{cc}
1 & \lambda_{i} \\
0 & 1
\end{array}\right)
$$

is a stubborn element of

$$
B=\left(\begin{array}{ll}
* & * \\
0 & *
\end{array}\right) \cap E(2, \mathcal{O})
$$

Proof On page 189 of [6], Carter observes that

$$
\left(\begin{array}{cc}
t & 0 \\
0 & t^{-1}
\end{array}\right)
$$

can be written as a product of elementary matrices, for any invertible $t \in \mathcal{O}$, as follows. If $\lambda$ is any invertible element, we may write

$$
\left(\begin{array}{cc}
0 & \lambda \\
-\lambda^{-1} & 0
\end{array}\right)=\left(\begin{array}{ll}
1 & \lambda \\
0 & 1
\end{array}\right)\left(\begin{array}{cc}
1 & 0 \\
-\lambda^{-1} & 1
\end{array}\right)\left(\begin{array}{ll}
1 & \lambda \\
0 & 1
\end{array}\right)
$$

and then note that

$$
\left(\begin{array}{cc}
t & 0 \\
0 & t^{-1}
\end{array}\right)=\left(\begin{array}{cc}
0 & t \\
-t^{-1} & 0
\end{array}\right)\left(\begin{array}{cc}
0 & -1 \\
1 & 0
\end{array}\right)
$$

Carter's observation shows that

$$
\left(\begin{array}{cc}
\omega & 0 \\
0 & \omega^{-1}
\end{array}\right)
$$

is in $E(2, \mathcal{O})$ for any unit $\omega$ in $\mathcal{O}$. Computing the commutator of

$$
\left(\begin{array}{cc}
\omega & 0 \\
0 & \omega^{-1}
\end{array}\right) \text { and }\left(\begin{array}{ll}
1 & \lambda \\
0 & 1
\end{array}\right)
$$

for $\omega$ a unit of $R$ and $\lambda \in R$ yields:

$$
\left[\left(\begin{array}{cc}
\omega & 0 \\
0 & \omega^{-1}
\end{array}\right),\left(\begin{array}{ll}
1 & \lambda \\
0 & 1
\end{array}\right)\right]=\left(\begin{array}{cc}
1 & \left(1-\omega^{2}\right) \lambda \\
0 & 1
\end{array}\right) .
$$

By assumption, the group of units of $\mathcal{O}$ is infinite. Dirichlet's units theorem (see, eg [28, Appendix B]) implies that we may choose $\omega_{0} \in \mathcal{O}^{*}$ a unit of infinite order. Let $H<B$ be a subgroup of index at most two. Then $H$ must contain

$$
\left(\begin{array}{cc}
\omega_{0}^{2} & 0 \\
0 & \omega_{0}^{-2}
\end{array}\right) \text { and }\left(\begin{array}{cc}
1 & 2 r \\
0 & 1
\end{array}\right)
$$

Algebraic 83 Geometric Topology, Volume 8 (2008) 
for all $r \in \mathcal{O}$. It follows from the computations (11) and (12) that

$$
\left(\begin{array}{ll}
1 & i \\
0 & 1
\end{array}\right) \in[H, H]
$$

for all $i$ in the ideal $I$ generated by $2\left(1-\omega_{0}^{2}\right)$. Let $N$ be the order of $R / I$. (The number $N$ is also called the norm of $I$; that it is finite when $I \neq(0)$ is an elementary fact of algebraic number theory; see, eg, [28, Chapter 5].) For any of the $\lambda_{i}$, we have

$$
\left(\begin{array}{cc}
1 & \lambda_{i} \\
0 & 1
\end{array}\right)^{N}=\left(\begin{array}{cc}
1 & N \lambda_{i} \\
0 & 1
\end{array}\right) \in[H, H]
$$

and so $\quad\left(\begin{array}{cc}1 & \lambda_{i} \\ 0 & 1\end{array}\right)$

is stubborn.

It remains to observe that $B<E(2, \mathcal{O})$ is solvable, and hence amenable. We may now apply Theorem 6.5 to conclude that $E(2, \mathcal{O})$ has Property $(\mathrm{QFA})$.

Remark 6.10 It was already known [24, page 68] that the groups covered by Theorem 6.8 possessed property (FA).

Remark 6.11 Another proof of Theorem 6.8 may be given as follows: First show that every unipotent is distorted. It follows that the bounded generators cannot (quasi)-act hyperbolically. It is shown in [18, Corollary 3.6] that there are no parabolic isometries of quasi-trees, and so each of the bounded generators (quasi)-acts elliptically. It then follows from bounded generation that any orbit is bounded.

Finally, we speculate on the variety of hyperbolic $\Gamma$-spaces, for $\Gamma=S L(2, \mathcal{O})$, where $\mathcal{O}$ is the ring of integers of a number field $k$. We have already remarked that $\Gamma$ is a lattice in

$$
\prod_{i=1}^{s} S L(2, \mathbb{R}) \times \prod_{i=1}^{t} S L(2, \mathbb{C}),
$$

where $s$ and $t$ are the number of real and complex places respectively. Projection to some factor gives an isometric action either on $\mathbb{H}^{2}$ or $\mathbb{H}^{3}$. Call a hyperbolic $\Gamma$-space standard if it is equivalent to $\mathbb{H}^{2}$ or $\mathbb{H}^{3}$ with one of these actions.

Conjecture 6.12 Every quasi-action by $\Gamma$ on a Gromov hyperbolic metric space either has an invariant horoball or is standard. 


\section{References}

[1] E Abe, Chevalley groups over local rings, Tôhoku Math. J. (2) 21 (1969) 474-494 MR0258837

[2] M Bestvina, K Fujiwara, Bounded cohomology of subgroups of mapping class groups, Geom. Topol. 6 (2002) 69-89 MR1914565

[3] M R Bridson, A Haefliger, Metric spaces of non-positive curvature, Grundlehren series 319, Springer, Berlin (1999) MR1744486

[4] M Burger, N Monod, Continuous bounded cohomology and applications to rigidity theory, Geom. Funct. Anal. 12 (2002) 219-280 MR1911660

[5] D Carter, G Keller, Bounded elementary generation of $\mathrm{SL}_{n}(\mathcal{O})$, Amer. J. Math. 105 (1983) 673-687 MR704220

[6] R W Carter, Simple groups of Lie type, Pure and Applied Math. 28, John Wiley \& Sons, London-New York-Sydney (1972) MR0407163

[7] C Chevalley, Sur certains groupes simples, Tôhoku Math. J. (2) 7 (1955) 14-66 MR0073602

[8] M Coornaert, T Delzant, A Papadopoulos, Géométrie et théorie des groupes: Les groupes hyperboliques de Gromov, Lecture Notes in Math. 1441, Springer, Berlin (1990) MR1075994

[9] K Fujiwara, The second bounded cohomology of a group acting on a Gromovhyperbolic space, Proc. London Math. Soc. (3) 76 (1998) 70-94 MR1476898

[10] M Fukunaga, Fixed points of elementary subgroups of Chevalley groups acting on trees, Tsukuba J. Math. 3 (1979) 7-16 MR561842

[11] T Gelander, A Karlsson, G A Margulis, Superrigidity, generalized harmonic maps and uniformly convex spaces, Geom. Funct. Anal. 17 (2008) 1524-1550 MR2377496

[12] M Gromov, Hyperbolic groups, from: "Essays in group theory", Math. Sci. Res. Inst. Publ. 8, Springer, New York (1987) 75-263 MR919829

[13] D Groves, J F Manning, Dehn filling in relatively hyperbolic groups, to appear in Israel J. Math. arXiv:math/0601311v3

[14] J E Humphreys, Introduction to Lie algebras and representation theory, Graduate Texts in Math. 9, Springer, New York (1978) MR499562 Second printing, revised

[15] A Karlsson, G A Noskov, Some groups having only elementary actions on metric spaces with hyperbolic boundaries, Geom. Dedicata 104 (2004) 119-137 MR2043957

[16] D Kotschick, Quasi-homomorphisms and stable lengths in mapping class groups, Proc. Amer. Math. Soc. 132 (2004) 3167-3175 MR2073290

[17] J F Manning, Geometry of pseudocharacters, Geom. Topol. 9 (2005) 1147-1185 MR2174263 
[18] J F Manning, Quasi-actions on trees and property (QFA), J. London Math. Soc. (2) 73 (2006) 84-108 MR2197372 With an appendix by N Monod and B Rémy

[19] H Matsumoto, Sur les sous-groupes arithmétiques des groupes semi-simples déployés, Ann. Sci. École Norm. Sup. (4) 2 (1969) 1-62 MR0240214

[20] N Monod, Superrigidity for irreducible lattices and geometric splitting, J. Amer. Math. Soc. 19 (2006) 781-814 MR2219304

[21] N Monod, Y Shalom, Cocycle superrigidity and bounded cohomology for negatively curved spaces, J. Differential Geom. 67 (2004) 395-455 MR2153026

[22] D W Morris, Bounded generation of $\operatorname{SL}(n, A)$ (after D Carter, G Keller, and E Paige), New York J. Math. 13 (2007) 383-421 MR2357719

[23] L Mosher, M Sageev, K Whyte, Quasi-actions on trees. I. Bounded valence, Ann. of Math. (2) 158 (2003) 115-164 MR1998479

[24] J-P Serre, Trees, Springer Monographs in Math., Springer, Berlin (2003) MR1954121 Translated from the French original by J Stillwell, Corrected 2nd printing of the 1980 English translation

[25] P B Shalen, Representations of 3-manifold groups, from: "Handbook of geometric topology", North-Holland, Amsterdam (2002) 955-1044 MR1886685

[26] M R Stein, Generators, relations and coverings of Chevalley groups over commutative rings, Amer. J. Math. 93 (1971) 965-1004 MR0322073

[27] R Steinberg, Lectures on Chevalley groups. Notes prepared by J Faulkner and R Wilson (1968)

[28] I Stewart, D Tall, Algebraic number theory and Fermat's last theorem, third edition, A K Peters Ltd., Natick, MA (2002) MR1876804

[29] O I Tavgen', Bounded generability of Chevalley groups over rings of $S$-integer algebraic numbers, Izv. Akad. Nauk SSSR Ser. Mat. 54 (1990) 97-122, 221-222 MR1044049

Dept of Mathematics, SUNY Buffalo

Buffalo, NY 14260-2900, USA

j399m@buffalo.edu

http: //www . math. buffalo.edu/ j399m

Received: 20 April 2007 